\title{
Relativisme et sociologie : le cas du sentiment de justice
}

Jean Kellerhals

\section{OpenEdition}

Journals

Édition électronique

URL : http://journals.openedition.org/ress/545

DOI : $10.4000 /$ ress. 545

ISSN : 1663-4446

Éditeur

Librairie Droz

Édition imprimée

Date de publication : 1 septembre 2003

Pagination : 137-150

ISBN : 2-600-00878-0

ISSN : 0048-8046

Référence électronique

Jean Kellerhals, "Relativisme et sociologie : le cas du sentiment de justice », Revue européenne des sciences sociales [En ligne], XLI-126 | 2003, mis en ligne le 30 novembre 2009, consulté le 19 avril 2019. URL : http://journals.openedition.org/ress/545; DOI : 10.4000/ress.545 
Jean KELLERHALS

\section{RELATIVISME ET SOCIOLOGIE: LE CAS DU SENTIMENT DE JUSTICE}

\section{INTRODUCTION}

L'évocation des normes et idéaux de justice présents dans les mentalités contemporaines est une manière privilégiée d'aborder la question du relativisme en sociologie. On peut en effet se demander, à propos d'une valeur - la justice dont on trouve partout l'expression, si la définition qu'en donnent les mentalités populaires est unique, généralisée, ou si au contraire cette exigence de justice trouve autant de formulations différentes qu'il y a de personnes. Ou encore chercher à voir si la grande variété des conceptions en ce domaine se résume finalement à un faible nombre de types, eux-mêmes associés à des structures sociales bien définies.

J'aimerais donc dans ces pages examiner d'abord, sur la base d'études empiriques, quelles «images du juste» prévalent aujourd'hui en matière de justice distributive - qui a droit à quelle gratification pour quelle prestation? - et dans le domaine pénal: quelles sanctions pour quel crime ou délit? J'aimerais ensuite cerner les facteurs essentiels de variation de ces images, pour conclure en risquant certaines hypothèses sur la question de l'universalisme ou du relativisme en ce domaine ${ }^{1}$.

Le choix de ce thème a peut-être d'autant plus de pertinence que le « débat de justice » s'est, sous l'action de quelques causes bien identifiables, beaucoup intensifié ces dernières décennies. Même si, nous dit Dumont (1967, 1985), la sensibilité universelle aux questions de justice est d'autant plus vive que l'on passe de sociétés fondées sur le double principe de hiérarchie et de destin - où des ordres, des castes, se voient attribués des droits et privilèges fondés sur la nature des choses plutôt que sur le mérite individuel - aux sociétés dites démocratiques, fondées sur la double idée de mérite et d'égalité. Et même si Tocqueville avait bien raison de relever que, paradoxalement, l'égalisation des conditions entraîne une virulence plus grande, plutôt qu'une accalmie, du débat de justice (1986). Il reste que ce dernier s'exacerbe d'autant plus, dans les sociétés dites démocratiques, que le rythme de variation des ressources disponibles est rapide. Or l'après-guerre a vu se télescoper les Trente glorieuses, les crises et chômages,

Ce texte est largement basé sur l'étude publiée sous le titre J. Kellerhals «Normes et idéaux de justice dans les mentalités contemporaines », in Kellerhals J., Manai D. et Roth R. (eds) Pour un droit pluriel, Helbing et Lichtenhahn, Faculté de Droit, Genève, 2002, pp. 339-354. Il reprend les idées exposées lors du Colloque «Sociologie et relativisme» tenu à Genève en l'honneur du professeur Jacques Coenen-Huther. 
l'essoufflement de l'Etat dit de providence, posant à chacun de ces spasmes la question des bénéficiaires et des laissés pour compte de l'opération.

Plus encore, la question de justice est avivée par la transformation des identités sociales des personnes, car celle-ci suppose une redéfinition de droits et de devoirs qui semblaient bien acquis. Or a cet égard, trois phénomènes majeurs ont marqué les dernières décennies:

Femmes et hommes ont vu leurs rôles respectifs et leurs destins professionnels se modifier profondément, se rapprocher, favorisant ainsi des comparaisons jugées naguère sacrilèges ou ridicules.

Le caractère structurel des migrations internationales, leur ampleur, associées à la mondialisation des échanges ont amené des interrogations cruciales sur le rapport complexe entre différence, citoyenneté et égalité des droits. Elles ont bouleversé du même coup le problème de la «communauté de justice » (Jacquemain, 1995), c'est-à-dire la question de savoir comment définir les frontières des territoires sur lesquels doivent régner les normes ou idéaux de justice que nous revendiquons pour nous ${ }^{2}$.

Dans les relations «familiales», l'ampleur des divorces, la généralisation des unions sans mariage, la montée des familles à quatre générations et les recompositions familiales ont exacerbé la question du juste: comment répartir les salaires, à qui vont les pensions, qui doit s'occuper des aînés, quand s'arrêtent les devoirs des parents, etc. Tout d'un coup, dans des domaines aussi cruciaux pour l'équilibre personnel, les modèles font défaut. Il faut compter, comparer. Exercice compliqué, mais culpabilisant aussi, dans la mesure où nos idéaux culturels nous interdisent presque de compter quand on s'aime.

C'est dire que les «routines de justice» se sont pour beaucoup défaites et que l'on se demande anxieusement comment reconstruire. Sur quels critères se baser? La philosophie sociale et politique, consciente du problème, a récemment élaboré nombre de conceptualisations, mais elles s'affrontent plutôt qu'elles ne convergent. C'est peut-être pourquoi, dès les années soixante, la théorie et l'empirie sociologique se sont aussi confrontées à cette question, en cherchant notamment à expliciter les formes contemporaines du sentiment de justice, avec peut-être l'espoir d'en dégager - de manière inductive plutôt que déductive - une norme générale qui permette ou guide l'intervention socio-juridique tout en résorbant le conflit.

\section{LE SENTIMENT DE JUSTICE: UNE NORME GENERALE?}

En tout cas, les premières théorisations «opérationnelles » des années soixante allaient clairement dans ce sens. C'est notamment tout l'effort de Homans et de la théorie de l'Equité (Walster E., Walster G. W et Berscheid E, 1978; Messick D. M. et Cook K. S., 1993) qui cherchent à démontrer qu'il y a impression de justice

Cf. les travaux de M. Walzer; sans nous rallier aux thèses de cet auteur, rappelons qu'il développe l'idée de variations des idéaux de justice selon les cultures dans Walzer M. : Spheres of Justice. A Defense of Pluralism and Equity, New York, Basic Books. 
lorsque les acteurs en présence sont gratifiés proportionnellement à leurs mérites. Dans cette perspective, si la répartition concrète des efforts et des ressources s'éloigne de cet équilibre, les lésés se sentent frustrés et tendent à le rétablir, les nantis se sentent coupables et prennent tous les prétextes pour diminuer la qualité ou la valeur des efforts des lésés. Il y a recherche d'un équilibre. Cette norme - qui est en somme celle de l'égalité de traitement - est, selon ces auteurs, universelle et unique. C'est donc une vision comparatiste de la justice: vivre la justice, c'est être traité comme les autres. Mais cet optimisme, qui voit apparemment le fait déterminer le contenu de la norme, n'a guère résisté aux analyses subséquentes.

D'abord, cette conception comparatiste de la justice apparaît, note Sampson (1980), comme très située historiquement. Dans des contextes moins marqués par la sécularisation, le sentiment de justice se donne comme plus ontologique: il est souvent un débat entre Dieu, la personne et sa destinée plutôt qu'entre des individus comparant leurs sorts respectifs.

Même si l'on s'en tient à une perspective comparatiste, il faut bien voir que cette comparaison implique, sauf dans des situations artificiellement simplifiées, la mise en jeu d'attributions sociales des causes, de sélection des unités comparées, de hiérarchisation des ressources, de frontières des échanges considérés, etc., qui sont non pas des «donnés» objectifs mais des représentations sociales construites (des «bonnes raisons » au sens de Boudon) (1995) inégalement distribuées ou formulées selon les acteurs en présence. Autrement dit, même si l'on est d'accord sur l'importance de la notion de mérite, il reste à définir celui-ci, et les avis des acteurs sur ce point sont souvent diamétralement opposés. Le « débat de justice» consiste alors à se demander comment s'opère cette construction sociale des mérites plutôt qu'à affirmer l'importance de ceux-ci.

Dès que l'on introduit la durée dans l'analyse de l'échange et du partage, on est inévitablement confronté, comme le montrent dans des registres différents Dupuy $(1986,1994)$, mais aussi Nozick ou van Parijs, au paradoxe des contradictions internes du principe d'égalité de traitement: en effet, mettre en œuvre le principe d'égalité des chances ou des efforts se traduit par une inégalité des satisfactions, garantir les droits acquis se paye par une inégalité des chances, etc. Ainsi le principe nécessaire d'égalité engendre-t-il autant de frustrations qu'il ne régule la compétition.

Les jugements de justice observés «in vivo» (Shepelak N. J et Alwin J. F., 1986; Alves W. et Rossi P. H., 1978), par exemple en matière de salaires, font de fait intervenir, selon des quantités variables, plusieurs principes de justice: le mérite, certes, qui paye chacun en fonction de ses efforts, mais aussi le principe du besoin, qui nomme juste une situation caractérisée par l'égalité des satisfactions même si les mérites sont inégaux et le principe d'égalité, qui veut que l'on ait les mêmes droits quels que soient nos mérites ou nos besoins. Cela reflète probablement le fait, bien analysé par Brickman (1981), Hochschild ou Törnblom (1983), que deux problèmes sont étroitement imbriqués dans les jugements de justice: comment assurer à chacun son dû, et comment garantir une régulation sociale qui permette le maintien de la production des ressources distribuables.

De surcroît, les travaux de Lerner nous montrent que la légitimité reconnue à telle ou telle norme dépend de la proximité des personnes en présence, de la durée de leurs échanges et de la diversité de ces derniers. Pour le dire (trop) simplement, une grande proximité d'autrui semble légitimer la mise en œuvre du principe du 
besoin, alors qu'un plus grand éloignement appelle la justification du principe du mérite. Dans le même esprit, plus autrui est défini de manière vague et abstraite, plus le mérite apparaît légitime, alors que l'on est davantage sensible au besoin lorsque cet autrui a un visage précis, identifiable. Dans cette perspective, établir la justice revient d'abord à établir (ou définir) quelle relation on a avec autrui - estce un frère, un étranger, un ennemi? - plutôt qu'à comptabiliser les mérite. La norme de justice est symbole d'une relation sociale qu'en retour elle contribue à renforcer.

Enfin les travaux de Deutsch ont beaucoup généré d'hypothèses sur le caractère finaliste de la norme de justice. L'idée est ici que, selon que le groupe considéré vise prioritairement la productivité plutôt que la cohésion ou encore la satisfaction de ses membres, il légitimera une norme de mérite plutôt qu'un critère d'égalité ou de besoin.

Il est donc à la fois impossible de conclure à l'existence d'une seule norme concrète de justice et assez artificiel de réduire les jugements de justice à l'application de telle ou telle norme spécifique - tels que l'égalité, le besoin, etc. - sans voir que la légitimité qui leur est reconnue dans telle ou telle situation repose en fait sur des représentations sociales, ou des visions du monde, plus larges, et notamment sur des conceptions de la responsabilité - civile et pénale - sur lesquelles j'aimerais maintenant centrer mon analyse.

\section{LES IMAGES DU JUSTE : QUELQUES OBSERVATIONS}

Analyser ces conceptions de la responsabilité revient à cerner comment les mentalités contemporaines élaborent les réponses à quatre questions fondamentales de régulation sociale bien connues du droit civil: celle de a) l'imputation: qui est responsable d'un acte?, b) celle de l'évaluation: comment mesurer l'ampleur des gains ou des pertes et selon quels critères les répartir?, c) celle de l'intervention de l'Etat: jusqu'où peut-il orienter les choix individuels?, et d) celle des assurances: dans quelles conditions doivent-elles recouvrir les responsabilités des acteurs? Les travaux que nous avons menés sur ce thème tant dans le domaine de la famille, que du contrat, de la responsabilité civile au sens large et de la sanction pénale montrent que les mentalités populaires abritent trois genres de conceptions de la responsabilité, et par-là de la justice, assez différentes et récurrentes malgré les contextes assez divers où on tente de les dégager.

S'agissant d'abord des représentations subjectives de la responsabilité civile, nos divers travaux, et notamment les recherches menées avec J.-F. Perrin sur «le juste contrat $»^{3}$, montrent que ces trois conceptions peuvent se laisser brièvement - allusivement - décrire ainsi :

a) Le providentialisme est dominé par une conception de l'individu vu comme jouet de forces sociales impersonnelles qui s'exercent sur lui. Ses comportements

Les types que l'on distingue ici sont construits à partir de plusieurs recherches présentées notamment dans Kellerhals J., Modak M., Perrin J.-F. et Sardi M. (1993). 
- de consommation, de risque, de travail, etc., traduisent l'importance du stress, de la compétition ambiante, de la publicité, de la hiérarchie. Dès lors, la justice consiste à le protéger contre tout. Il est légitime de lui reconnaître le droit de se dédire, sans motif, des engagements - achats ou autres - qu'il a contractés: il a un «droit au regret» généralisé. S'il commet des fautes importantes et néanmoins évitables dans l'entreprise qui l'emploie, c'est à cette dernière de payer les dégâts : il n'est que l'agent d'une volonté qui lui est supérieure. Il ne devra par ailleurs réparer les dégâts dus à ses imprudences éventuelles dans la vie quotidienne que dans la mesure de ses disponibilités personnelles, et non pas à la hauteur objective ou marchande de ces dégâts. Les assurances devront le protéger même en cas de faute grave (ivresse au volant par exemple). Réciproquement, on s'attend à ce que les entreprises assument les dégâts causés par leurs activités ou produits, même si elles ne pouvaient pas les prévoir ou qu'ils résultent d'une utilisation inadéquate. L'idée de responsabilité du fait du produit est donc poussée ici à l'extrême. Cette protection généralisée de l'individu ne s'encombre guère de contraintes : c'est à la personne de choisir les risques qu'elle prend (sports extrêmes, drogues, endettement, etc.) et non pas à l'Etat de définir les marges de sa liberté. Celui-ci par contre devra au besoin prendre le relais pour dédommager la personne si celle-ci, par malheur, est victime d'agressions dont les fautifs ne sont pas identifiables («transférabilité» de la responsabilité). Enfin, et c'est essentiel, les souffrances psychologiques dont la personne est éventuellement victime peuvent et doivent être compensées par d'importantes contreparties matérielles: il y a en somme convertibilité générale des ressources et des affects.

b) Une deuxième conception - le volontarisme - met par contre en exergue l'importance décisive de la volonté individuelle pour la formation et l'assomption des responsabilités. Dans cette perspective, malgré les influences de toutes sortes, c'est la personne qui choisit en définitive de fumer, de s'endetter, de foncer au volant. Elle doit donc assumer les conséquences de ces choix. Le droit au regret rendre un ordinateur que l'on vient d'acheter, ne pas honorer une réservation si l'on est malade, etc. - n'est pas reconnu sauf circonstances extrêmes. De surcroît, la réparation des erreurs commises par un employé dans l'entreprise qui l'emploie doit être à la charge de celui-ci si la faute était évitable. Les dommages commis doivent être réparés à leur hauteur réelle, et non pas en fonction des moyens subjectifs de la personne. La place laissée à la compensation financière du tort moral est mince, parce qu' on ne peut pas raisonnablement compenser un deuil par de l'argent (refus de la «convertibilité»). Enfin, c'est au fautif de réparer un dégât: cela signifie que l'Etat n'a pas du tout le devoir de réparer des pertes que des inconnus ou des personnes insolvables ont causées à un tiers. De même l'Etat doit-il sauf exceptions laisser la personne libre de s'assurer ou non contre les risques qu'elle voudra prendre. Il s'agit de sa liberté.

c) Une troisième perspective peut être dite communautarisme en ceci qu'elle est fondée sur la pesée des intérêts comparés des parties en cause, qui fournit le critère essentiel de justice. L'idée est qu'en principe la personne est titulaire de ses actes, mais qu'il existe fréquemment des circonstances où les pressions qui s'exercent sur elle sont telles qu'elles légitiment une exonération partielle ou totale de sa responsabilité. Concrètement, le droit au regret existe - pour un achat, 
un bail, etc. - mais seulement si la personne peut invoquer de bons motifs (maladie soudaine, changement de situation, etc.). L'idée est que l'exécution du contrat, en soi légitime, ne doit cependant pas placer la personne dans des situations impossibles, ou provoquer des effets néfastes sans rapport avec les gains du titulaire des droits. C'est dire aussi que les réparations dues au titre d'un dommage le sont à hauteur des moyens de la personne et non à la hauteur des dégâts euxmêmes. Ou encore que l'agentisme - le fait que l'entreprise paie pour les fautes de l'employé - est accepté, même s'il n'y a pas erreur grave et évitable de ce dernier, simplement parce que l'entreprise est en principe plus forte. Toujours dans cette perspective, les réparations pour tort moral sont surtout symboliques: on ne peut échanger de l'inconfort psychologique, de l'affliction, contre de l'argent. Du point de vue des tâches de l'Etat, ce dernier a le devoir de réparer les préjudices provoqués par des individus non identifiés ou non solvables: «transférabilité » de la responsabilité. Par contre, l'Etat a le droit d'exiger que la personne s'assure contre les risques les plus courants et les plus graves et aussi celui de limiter les risques extrêmes que la personne peut prendre. Est donc dite juste, dans cette troisième perspective, une décision qui vise le bien-être ultime des personnes et des groupes plutôt qu'un choix qui respecte des créances ou les volontés.

S'agissant ensuite des représentations de la justice pénale, l'étude que nous avons menée avec C. N. Robert, N. Languin et E. Widmer (2001) permet aussi de dégager trois genres de philosophies populaires dont les analogies avec les conceptions de la responsabilité civile sont frappantes. a) Le finalisme - très proche du communautarisme évoqué il y a un instant - justifie principalement la sanction pénale par sa vertu supposée de réinsertion de l'individu dans la société. Certes, il faut punir, mais le but premier de la sanction est de réintégrer. Le délinquant est perçu essentiellement comme une victime de son parcours de vie: famille perturbée, relations abîmées, exclusion économique et sociale - plutôt que comme doté de mauvais penchants ou de tares acquises. Les réponses aux infractions sont modérées, peu inspirées par l'esprit de vengeance et tournées vers l'amendement par le soin. Même les souffrances de la victime ne sont guère prises en compte pour moduler la sanction.

b) Le contractualisme - très proche du volontarisme évoqué ci-dessus - met au contraire en avant la responsabilité personnelle du délinquant: il doit avant tout payer pour ce qu'il a fait. Qu'il ait eu une enfance difficile est secondaire, comme est secondaire l'éventuel objectif d'insertion sociale. La cause de la délinquance n'est pas à chercher dans l'exclusion ou la marginalité socio-économique, mais dans un contexte éducatif primaire qui n'a peut-être pas suffisamment armé moralement le jeune ou dans un calcul coût/bénéfice utilitaire qui fait préférer l'infraction à la conformité. La finalité de la peine est dominée par l'idée de restitution, de réparation due à la victime comme à la société. Dans cette optique volontariste, la sanction doit être surtout modulée par l'intention de l'auteur et la gravité objective des dégâts.

c) L'ostracisme enfin base ses critères de juste sanction sur une image très statutaire, catégorielle, du criminel : celui-ci est souvent appréhendé à travers des catégories comme l'étranger, ou le malade mental, ou le drogué. Il est d'abord autre. La vision de la criminalité est celle d'un phénomène en hausse, hausse due 
pour l'essentiel à des groupes ou agrégats sociaux précis. Est mauvais celui qui est autre. La vision quantitative de la délinquance est ici très irréaliste: dans l'imaginaire de ces personnes, les prétoires voient se bousculer surtout des meurtriers, des délinquants sexuels, de grands bandits. La justice consiste alors à les exclure. Ce n'est pas la réinsertion du fautif qui est visée ici, mais son expulsion définitive. Autrement dit, la sévérité de la sanction est très forte, et modulée par les appartenances - le statut - de l'accusé. On n'exclut pas l'utilisation de sanctions infamantes ou honteuses. Il serait assez aisé de montrer - mais je n'ai pas la place de développer vraiment ce point - que cet ostracisme est paradoxalement l'image inversée du providentialisme évoqué plus haut. Le principe mis en oeuvre dans les deux cas est en effet pareil: fonder la justice sur un «statut social»-tout bon, tout mauvais - de la personne. C'est-à-dire: a) faire équivaloir le juste à la définition de mérites (positifs ou négatifs) et b) déduire ces mérites de l'appartenance de la personne à une catégorie collective.

Enfin, les formes de sentiments de justice que nous avions pu mettre à jour dans nos analyses du fonctionnement familial (Kellerhals J., Coenen-Huther J. et Modak M., 1988) recoupent assez bien les types que l'on vient d'esquisser. En effet, les époux ou compagnons que l'on a pu observer utilisent trois perspectives différentes pour juger de la justice de leurs échanges ou de leur partage:

a) Une première manière de juger peut être dite «statutaire»: telle répartition des charges ou des droits apparaît juste parce qu'elle est semblable à celle qui prévaut chez les autres personnes de même statut (par exemple les autres ménagères, les autres adolescents, les autres grands-parents, etc.). C'est donc une vision comparatiste et catégorielle de la justice, légitimée le plus souvent de manière «traditionnelle» (au sens de M. Weber), c'est-à-dire en faisant appel à des déterminations biologiques ( «les femmes sont généreuses», «les hommes n’y pensent pas» ou historiques («c'est le rôle des aînés, on a toujours fait comme ça»). On voit donc bien la proximité de principe avec ce que l'on vient d'évoquer à propos de l'«ostracisme».

b) Une deuxième manière de juger est «finaliste »: est dit juste un état de faits qui est censé produire des conséquences heureuses pour le groupe ou la personne. Par exemple, on estimera qu'il est juste que les enfants reçoivent le même montant d'argent de poche parce que «cela limite les conflits, les jalousies »; ou qu'il est bon que chacun d'eux ait sa part des travaux domestiques «parce que ça leur apprend que, dans la vie, il faut faire des efforts ». Ce n'est donc pas une perspective comparatiste, ni un savant équilibre de créances objectives qui sert de critère, mais un «bien» ultime qui transcende tant les dettes que les statuts. Le juste est l'instrument du bien.

c) Enfin, la troisième perspective est de type «contractualiste»: la répartition des efforts et des gratifications dans la famille est dite juste si elle correspond à un accord explicite entre les personnes concernées. Chacun a été consulté, a pu s'exprimer, dire ses besoins et ses envies. Dès lors, le résultat est juste. C'est en quelque sorte une conception procédurale de la justice qui prévaut ici. Cette dernière manière de penser est évidemment voisine de ce que l'on a envisagé plus haut sous les étiquettes de volontarisme et de contractualisme. 
Ce bref rappel montre bien non seulement que les jugements de justice trouvent leur place dans des relations très personnelles et intimes autant que dans des relations marchandes, mais aussi que les catégories de jugement utilisées dans ces deux contextes y sont qualitativement comparables (même si, bien sûr, les quantités ou les distributions statistiques diffèrent).

\section{L'ANCRAGE DES IMAGES}

Face à cette pluralité des images du juste, on peut alors se demander quels facteurs les modèlent et si l'on peut faire certaines conjectures (forcément fragiles) sur l'évolution de ces conceptions. On peut regrouper ces facteurs sous deux registres ou thèmes importants: le pouvoir et l'appartenance.

\section{A. Le pouvoir}

En matière de représentations subjectives de la responsabilité civile, on relèvera en premier lieu que la légitimité accordée au providentialisme plutôt qu'au volontarisme dépend beaucoup des personnes impliquées dans les problèmes soumis à jugement. S'il s'agit de deux personnes physiques clairement identifiables, le volontarisme est davantage revendiqué. S'il s'agit au contraire d'une personne physique qui fait contrat ou s'attaque à une organisation anonyme, le providentialisme est à l'inverse plus souvent légitimé. Or comme les interactions d'individus avec de grandes institutions ou organisations tendent aujourd'hui à se généraliser beaucoup - dans le domaine de la consommation courante, des loyers, des baux de travail, des assurances etc. - on peut comprendre que les représentations providentialistes gagnent du terrain. Plus généralement, on voit qu'une grande dichotomie traverse la morale contractuelle contemporaine. Du côté des relations interpersonnelles, cette morale se veut centrée sur la personne. Elle est donc irréversible (les engagements sont les engagements), symétrique (droits et devoirs sont identiques pour les deux parties) intentionnelle (c'est la volonté qui l'emporte sur tout autre critère). Du côté des relations avec les organisations, cette morale est au contraire réversible (on peut revenir sur les engagements), asymétrique (les droits de la personne sont davantage protégés que ceux de l'organisation) et non-intentionnelle (on n'est pas forcément responsable de ce que l'on a voulu ou provoqué; on ne doit réparation qu'en mesure de ses moyens).

En deuxième lieu, et s'agissant toujours de responsabilité civile, on relèvera avec Ewald que la généralisation des assurances a déplacé en partie le débat de justice. Alors que naguère la question portait surtout sur la question de l'imputation causale - qui a fait quoi - et que la réponse à cette question entraînait la définition des sanctions et récompenses, la place croissante des assurances donne une importance primordiale à la question de savoir qui peut - au sens d'être en mesure de - payer quoi, la réponse à cette question tendant à la fois à définir le responsable et à déterminer l'ampleur du dégât. Le débat de justice quitte alors en partie l'agora des citoyens pour se réfugier souvent dans les cénacles de spécialistes. Certes, la sécurité y gagne peut-être. Mais tout un chacun devient en quelque sorte étranger à la question de sa propre responsabilité. On observe alors une sorte de 
refuge du moralisme traditionnel dans la sphère privée - celle des amis, de la famille, de quelques proches - par différence à la place publique.

En filigrane de ces considérations, on voit bien que les positions de pouvoir «informent» les représentations du juste et les normes subjectives qui en découlent. Dans les diverses observations que nous avons pu faire - tant dans les familles qu'à propos des contrats ou de la responsabilité - comme dans la littérature analysée ${ }^{4}$ la légitimité donnée au volontarisme est proportionnelle au niveau de ressources culturelles ou économiques dont disposent les personnes. Fortement dotée, la personne se dit et se veut maîtresse de son destin, gestionnaire rationnelle de ses atouts. Elle sait qu'elle pourra y gagner, et toute son image de soi est basée sur l'importance de sa valeur comme être autonome. Réciproquement, l'absence de ressources est certes associée dans quelques bons cas à une mobilisation contestatrice, mais aussi, et plus souvent, liée selon les domaines à une légitimation plus grande du providentialisme ou à une caution plus nette pour l'ostracisme. L'absence de pouvoir favorise donc les conceptions de la responsabilité qui découplent individu et communauté, et tend à donner à la notion de catégorie comme les jeunes, les femmes, les étrangers, etc. - une importance déterminante. La personne disparaît derrière une surdétermination de ses droits, devoirs, privilèges ou tares liée à ses origines, ses appartenances. Attribuer du pouvoir aux démunis apparaîtrait donc, dans cette perspective, comme une bonne manière de lutter contre les dérives du sentiment de responsabilité.

\section{B. L'appartenance}

Le deuxième thème - celui de l'appartenance - s'inscrit dans le prolongement des travaux de Lerner (1977; Cohen R. L. et Greenberg J., 1982) (et bien d'autres auteurs après lui ${ }^{5}$ ) évoqués plus haut. En bâtissant sur ses propositions, on voit bien que l'accroissement - aujourd'hui - de la mobilité relationnelle dans les domaines de vie personnelle les plus décisifs - montée des divorces, généralisation des unions sans mariage, (mais aussi mobilité professionnelle, statuts précaires) etc. - favorise certainement une conception du juste caractérisée, pour reprendre le langage des ethnologues, par l'exigence de réciprocité immédiate. Il faut que tout se compense ou s'équilibre tout de suite, ou sur le même plan. Il y a donc fort à parier qu'un accroissement de la mobilité relationnelle et/ou l'incertitude pesant sur la stabilité du réseau des proches se traduise par des conceptions du juste où le don et la dette tiennent en fait une place moins grande, et cela au bénéfice de conceptions «comptables» de la justice et d'un certain affaiblissement du sentiment de solidarité intergénérationnelle ou intergroupe. L'observation ethnologique rejoint ici l'expérimentation psychosociologique: passer du durable au provisoire limite la légitimité de la norme du besoin au profit d'une vision plus contractuelle de la justice. Pour le dire dans les termes de Gilligan

Cf. par exemple, Hochschild A. (1981), What's Fair? American Beliefs about Distributive Justice, Cambridge Mass., Harvard University Press; Hamilton V. L. et Rytina S. (1980), «Social Consensus on Norms of Justice: Should the Punishment fit the Crime?», American Journal of Sociology, 85, pp. 1117-1144; Alves et Rossi (1978), op. cit.; Selnick P. (1969), Law, Society and Industrial Justice, New York, Russel Sage Foundation.

5 Par exemple, Carles et Carver, Greenberg, Tindale et Davis, etc. 
(1983): la légitimation d'une justice définie comme «caring» (l'«agapé » dans la nomenclature de Boltanski [1990]) risque de s'effacer quelque peu devant celle, plus masculine selon elle, d'une recherche de justice fondée sur le fait d'honorer des créances accumulées.

Plus généralement, l'incertitude pesant sur les appartenances - groupales, régionales, culturelles - complique énormément la question déjà évoquée de la "communauté de justice». A qui, pour reprendre les termes de Walzer, doit-on justice, à qui doit-on simplement entraide? Je n'entends pas, en évoquant cet auteur, reprendre à mon compte ses thèses et solutions. Mais il est clair que le flou des appartenances peut théoriquement aussi bien déboucher sur une mondialisation des préoccupations de justice que sur le repli frileux d' une solidarité bornée aux plus proches.

\section{CONVERGENCES}

Tentons une synthèse. Il existe probablement une constante anthropologique sous-tendant les divers avatars de l'exigence de justice que j'ai brièvement examinés: c'est que cette exigence porte moins sur l'équilibration de choses échangées que sur la «reconnaissance» de la personne. L'enjeu fondamental, c'est le respect, l'identité. Dans des perspectives très différentes, des penseurs comme Rawls (1972) - «qui place l'estime de soi parmi les plus importants des 'biens premiers' que la société est chargée de fournir»- ou comme van Parijs avec son idée «d'égale sollicitude ${ }^{6}$ peuvent peut-être se retrouver dans cette thèse. Mais cette reconnaissance de l'autre prend, à travers sociétés et situations, trois visages principaux. Dans certains cas, rendre justice, c'est avant tout viser un bien futur - de la personne ou du groupe. Est juste ce qui produit des conséquences heureuses. L'acteur est donc envisagé dans son projet, son devenir. Dans d'autres situations, rendre justice équivaut à reconnaître la volonté de l'acteur, valider son intention ou les actes qu'il a voulu poser. On est en quelque sorte dans le présent. Dans d'autres cas enfin, reconnaître la personne revient à valider ses origines, ses ancrages, voir en elle l'héritière d'une communauté et d'un statut. On est, si l'on peut s'exprimer ainsi, dans le passé.

Une justice idéale voudrait probablement que l'on parvienne à une reconnaissance de l'autre dans ces trois dimensions à la fois. C'est bien sûr très difficile, et je ne suis pas loin de penser que l'exigence de justice contemporaine est forcément malheureuse. En effet, s'agissant aussi bien de justice pénale que de justice distributive, on est frappé - par delà les différences d'accent rencontrées dans les types évoqués ci-dessus - par l'aspect à certains égards prométhéen des aspirations contemporaines en matière de justice. En matière pénale, rétablir la victime dans ses droits, protéger les valeurs fondamentales de la société et réintégrer le coupable apparaissent comme autant d'objectifs à poursuivre simultanément et qui, ensemble, légitiment la sanction. Mais en même temps, chacun sait bien que des peines très génériques comme la prison n'atteignent que mal ce triple objectif. Il y a donc une sorte de conscience malheureuse - une forme d'anomie - face à la

\footnotetext{
${ }^{6} \quad$ Cf. Jacquemain M., op. cit.
} 
sanction, qui est le pendant de ce que l'on observe en matière de justice distributive. Là aussi, il y a «trop plein »: récompenser les efforts, assurer la satisfaction des besoins de base, garantir la sécurité à long terme, laisser à chacun son autonomie apparaissent comme autant de critères de justice dont aucun n'est secondaire. De ce point de vue, nos sociétés ne se caractérisent pas, comme on le répète trop volontiers dans les cantines, par une perte des valeurs, mais bien par une volonté obstinée de les poursuivre toutes simultanément. Or il faut bien choisir, et ce choix est frustrant et conflictuel. Il l'est peut-être et surtout parce que le «tragique» - c'est-à-dire la nécessité, selon Elster, d'inclure dans certains choix de justice la négation radicale de certains droits élémentaires des personnes - est comme éliminé de nos conceptions de la justice, peut-être parce qu'elles ne peuvent donner à ce tragique aucun sens eschatologique.

C'est pourquoi à mon sens les sociétés contemporaines sont tentées par diverses manières de pervertir la reconnaissance des personnes. J'en évoquerai quatre, non exhaustives et inégalement importantes. La première, pour reprendre une idée de Sampson ${ }^{7}$, consiste à identifier le juste à la satisfaction du désir en considérant comme légitime toute aspiration individuelle. Une forme exacerbée de providentialisme qui verrait l'exaltation du désir succéder à la comptabilité des mérites, elle-même ayant détrôné la légitimité reconnue à des privilèges associés au statut. Le deuxième genre de perversion consiste à dire que chacun est responsable de son destin, qu'il se débrouille comme il l'entend, qu'il y a justice dès lors que les règles du jeu sont les mêmes pour tous, quand bien même chacun est très conscient de l'importance des déterminations sociales des aptitudes et des goûts.

Un troisième genre - peut-être parent du deuxième - consiste à répondre à la difficulté d'élaborer une consigne juste par la délégation du problème à des spécialistes - par exemple des médecins, des psychologues ou des conseillers sociaux, etc. - qui ne peuvent eux-mêmes que s'en remettre, faute de compétences adéquates, à la subjectivité de leurs clients ou patients. Etrange jeu de masques où les professionnels et les profanes se disqualifient mutuellement en donnant cependant l'impression de se reconnaître (Kellerhals J. et Pasini W., 1976).

Enfin, l'appel à la responsabilité individuelle, ou la «responsabilisation» ne reflète quelquefois, comme le montrent Messant et Modak $^{8}$, que la demande pressante et rituelle - faite aux employés d'un service ou d'une entreprise - il peut s'agir d'informatique comme de protection de la jeunesse - de prendre sur eux le poids d'une mission mal définie, ambivalente ou trop ambitieuse. La «responsabilité de diligence», plutôt que l'obligation de prestations définies, confine alors au mobbing et à la culpabilisation plutôt qu'à la « reconnaissance».

Dans tous ces cas, il s'agit de réduire au seul individu la dialectique entre individu et communauté.

Le caractère illusoire de ces extrêmes, allié à la diversité des images du juste et à la nécessité de construire une norme régulatrice conduit à accorder une attention particulière aujourd'hui aux questions de justice procédurale, c'est-à-dire aux conditions jugées acceptables par les acteurs pour parvenir à un jugement de

Op. cit.

$8 \quad$ Messant F. et Modak M.: «Un nouveau sens de l'engagement dans l'entreprise? La construction de la responsabilité chez les cols blancs des P.M.I.», Projet FNRS no 120466963.96. 
justice. La difficulté de construire une norme de justice unique, déductive et abstraite rend heureusement sa place au politique et donc aux conditions de la négociation. Nombre de travaux, après les percées de Thibaut et Walker (1975) ${ }^{9}$ ou de Leventhal, se penchent aujourd'hui sur ce thème. L'analyse des conceptions populaires de la justice procédurale montre à la fois que des normes récurrentes se dégagent, comme par exemple la nécessité a) d'entendre les personnes concernées, b) de mettre l'information pertinente à disposition, c) de définir et publier les procédures de décisions, les règles du jeu, d) d'organiser des voies d'appel, e) de garantir la neutralité et la compétence des experts. Mais elle montre aussi que ces procédures «fairs » ne sont elles-mêmes que des médiatrices assez approximatives de valeurs telles que le respect, l'empathie, l'honnêteté. Le danger serait donc, en matière de justice procédurale comme dans le domaine de la justice distributive, de réduire la reconnaissance de l'autre à des normes - certes absolument nécessaires, mais radicalement insuffisantes - qui ne sont en fait que des outils, des indicateurs prudentiels guidant le débat de justice mais ne se confondant pas avec celui-ci.

Ici encore, il faut élargir la question. Une société plurielle, ouverte et laïque trois caractères que nous valorisons énormément - a forcément pour rançon l'absence de critères «tout faits » et consensuels de justice et de responsabilité. Sauf à devenir une dictature bureaucratique, elle ne peut les créer d'un coup de baguette magique. Et pourtant, il faut faire des choix, construire des normes. Un remède certes partiel - à cette sorte de dilemme consiste à provoquer autant que possible l'interconnaissance et le dialogue. Autrement dit, créer du lien, partout et à tous les niveaux. Donner la parole. Quand la norme ne peut plus provenir du Dogme, elle doit surgir du lien. Le lien ajouté à la mixité produit l'invention, le progrès, alors qu'au contraire, l'anonymat associé à la mobilité et à la mixité crée l'indifférence, renforce les routines bureaucratiques et les solutions inadéquates.

Créer du lien, c'est favoriser cette reconnaissance d'autrui qui permet d'élaborer les critères de justice adéquats et d'aiguiser le sentiment de responsabilité. Mais, plus encore, créer du lien, c'est construire, concrétiser, cet Autrui envers qui on se doit d'être responsable. La difficulté des sociétés contemporaines à affronter la question de la responsabilité tient peut-être au fait que l'on ne sait plus bien quel est le visage d'autrui, ou envers qui l'on se veut loyal. Anonyme, autre, fugace, Autrui ne m'interpelle plus. Créer du lien permet alors de lier deux termes confondus dans l'expression française de responsabilité mais complémentaires en anglais - de «responsibility» (devoir quelque chose) et d' «accountability» (à quelqu'un). C'est, aujourd'hui, un enjeu majeur.

Je terminerai en émettant une hypothèse nourrie par la recherche sociologique empirique. Si la norme de justice apparaît comme l'expression symbolique d'une relation, la réciproque est souvent vraie. Cela signifie qu'en instaurant une solidarité objective entre les groupes et les personnes - fondée sur la reconnaissance de leurs besoins fondamentaux - on a de bonnes chances de promouvoir entre elles un sentiment de parenté, et - pourquoi pas - de fraternité. En mettant davantage

Thibaut J. et Walker L. (1975), Procedural Justice : A Psychological Analysis, Hillsdale, New York, Erlbaum. 
cette hypothèse en oeuvre dans leurs dessins de recherche, sociologues et psychologues sociaux pourraient construire efficacement le pont entre théorisation et intervention, et du même coup intensifier un fructueux dialogue entre droit et sociologie.

\section{Département de sociologie \\ Université de Genève}

\section{RÉFÉRENCES BIBLIOGRAPHIQUES}

Alves W. et Rossi P. H. (1978), «Who Should Get What? Fairness Judgements of the Distribution of Earnings », American Journal of Sociology, 84, pp. 541-563.

Boltanski L. (1990), L'amour et la justice comme compétences, Paris, Métailié, p. 119.

Boudon R. (1995), Le Juste et le Vrai, Paris, Fayard.

Brickman Ph., Folger R., Goode E., Schul Y., (1981), «Micro-Justice and Macro-Justice» in Lerner M. J. et Lerner S. C. (eds) The Justice Motive in Social Behaviour, New York, Plenum, pp. 173204.

Cohen R. L. et Greenberg J. (1982), «The Justice Concept in Social Psychology», in Greenberg J. et Cohen R. L. (eds) (1982), Equity and Justice in Social Behaviour, New York, Academic Press.

Dumont L. (1985), Homo Aequalis, Paris, Gallimard et Dumont L. (1967), Homo Hierarchicus, Paris, Gallimard.

Dupuy J.-P. (1994), «Les affaires sont les affaires», in La Justice, l'obligation impossible, Paris, Autrement, Série Morales; cf. aussi du même auteur (1986), La Justice sociale introuvable, Commentaire, 34, pp. 213-222.

Gilligan C. (1983), In a Different Voice. Psychological Theory and Women's Development, Cambridge Mass., Harvard University Press.

Hamilton V. L. et Rytina S. (1980), «Social Consensus on Norms of Justice: Should the Punishment fit the Crime?», American Journal of Sociology, 85, pp. 1117-1144.

Hochschild A. (1981), What's Fair? American Beliefs about Distributive Justice, Cambridge Mass., Harvard University Press.

Jacquemain M. (1995), «Représentation de la justice sociale. L'exemple de la Belgique francophone», L'Année Sociologique, 1995, 45, no 2, pp. 399-430.

Kellerhals J. et Pasini W. (1976), Le sens de l' avortement, Genève, Georg.

Kellerhals J., Coenen-Huther J. et Modak M. (1988), Figures de l' Equité, Paris, Presses Universitaires de France, coll. Le Sociologue.

Kellerhals J., Modak M., Perrin J.-F. et Sardi M. (1993), «L'Ethique du Contrat», Année Sociologique, 43, pp. 125-158.

Kellerhals J., Modak M. et Sardi M. (1995), «Justice, sens de la responsabilité et relations sociales », Année Sociologique, 45, no 2, pp. 317-349.

Kellerhals J., Modak M. et Perroquet D. (1997), Le sentiment de justice dans les relations sociales, Paris, Presses universitaires de France, «Que Sais-je».

Kellerhals J., Languin N., Sardi M., Lieberherr R. et Aeschimann G. (1998), «Les conceptions populaires de la responsabilité et du rôle des assurances: une étude des formes de justice et de solidarité dans les mentalités contemporaines », Rapport au FNRS no 1214- 046968.96.

Kellerhals J., Languin N. et al. (2000), «Formes du sentiment de responsabilité dans les mentalités contemporaines », Revue française de sociologie, avril-juin, 41-2, pp. 307ss.

Kellerhals J., Languin N. et al. (2001), «Le sentiment de responsabilité dans les mentalités contemporaines», Droit et Société, no 47, pp. 57ss. 
Lerner M. J. (1977), «The Justice Motive: Some Hypotheses as to its Origins and Forms », Journal of Personality, vol. 45, no 1, pp. 1-52.

Messant F. et Modak M. : «Un nouveau sens de l'engagement dans l'entreprise? La construction de la responsabilité chez les cols blancs des P.M.I.», Projet FNRS no 120466963.96.

Messick D. M. et Cook K. S. (1993), Equity Theory: Psychological and Sociological Perspectives, New York, Praeger.

Rawls J. (1972), A Theory of Justice, Oxford, Oxford University Press.

Robert C. N., Kellerhals J., Languin N., Widmer E., Poncela P., avec Boggio Y., Pattaroni L. et Coumanne C. (2001), «Les représentations sociales de la sanction pénale», Rapport FNRS 114.055893.98.

Sampson E. E. (1980), «Justice and Social Character», in Mikula (ed) (1980), Justice and Social Interaction, Wien, Huber, pp. 285-314.

Selnick P. (1969), Law, Society and Industrial Justice, New York, Russel Sage Foundation.

Shepelelak N. J. et Alwin J. F. (1986), «Beliefs about Inequality and Perceptions of Distributive Justice», American Sociological Review, 51, pp. 30-46.

Thibaut J. et Walker L. (1975), Procedural Justice: A Psychological Analysis, Hillsdale, New York, Erlbaum.

Tocqueville A. de, (1986, ed. or. 1840), De la Démocratie en Amérique, Paris, Laffont.

Törnblom K. Y. et Foa J. G. (1983), « Choice of Distribution Principle Cross Cultural Evidence of the Effects of Ressources », Acta Sociologica, 26.

Walster E., Walster G. W. et Berscheid E. (1978), Equity. Theory and Research, Boston, Allyn and Bacon.

Walzer M., Spheres of Justice. A Defense of Pluralism and Equity, New York, Basic Books. 